\title{
L'Hisloriographie de la Révolution Française a la veille du bicentenaire
}

\section{Michel Vovelle}

\section{La Révolution est-elle "terminée"?}

Qu'on me permette de débuter en rapportant une boutade, ma1s c'est une boutade amère: elle me renvore à ma première rencontre avec Albert Soboul, et c'étalt, si j'al bonne mémorre en 1958. Jeune agrégé d'historre, je m'engageais dans la voie de la recherche, avec l'idée de travaller sur la Révolution. Et Albert Soboul que je consultais, de me dire tout à trac Pourquoi veux-tu travailler sur la Révolution Française? Ça n'intéresse plus personne. Remarque paradoxale. luı-même, soutenant alors sa thèse, était au farte de sa productivité -- et, nous le verrons à l'instant, il était loin d'être seul, ou solé.

Force m'est pourtant de me remémorer cette phrase pessimiste, lorsque je considère combien, dans une optıque toute différente, l'ıdée a chemıné. Ce qu'exprime en termes d'humour inquiet Albert Soboul en 1958, bientôt Franço1s Furet le reprendra, définissant la Révolution comme un objet frold, puis annonçant en 1978 (Penser la Révolution) la Révolution est terminée, phrase qu'll commente en 1986 (Le Nouvel Observateur, 28 février) Quand j'al écrit la Révolution est terminée c'est une manière d'exprimer un voeu et un constat.

Ne soupçonnons pas Françors Furet d'arrières pensées homicides: mais lorsqu'il se place ainsi au niveau du souhait, 1 considère bien non seulement qu'une cértaine historiographie de la Révolution Françalse a vécu, mais plus encore que pour qu'une autre puisse renaitre - plus sereine, ou plus scientıfique sans doute - il convient bien que l'objet du débat soit revêtu selon l'expression consacrée de la beauté che mort.

Ce farsant, il ne fait que répercuter avec finesse une des nouvelles idées reçues, souvent formulée en termes plus banals. tout n'a-t-il pas été dit ou plutôt écrit sur la Révolution Françaıse? Quelles retouches apporter à un canevas événementıel sans mystère? $\mathrm{Ou}$, avec plus de perfidie, l'hıstoriographıe françaıse de la Révolution ne s'est-elle pas sclérosée, devenant répétitive, en s'enfermant dans le dogmatısme, quitte à laisser la fraicheur des nouvelles découvertes à d'autres, les anglo-saxons peut-être, dont on souligne l'actıvité sur ce chantier qu'lls abordent sans préjugés.

Entre les constats et les procès d'intention, quelques réalités s'imposent, au tournant des années 80 , dans l'appréciation de ce paysage collectif. Comme elle a reculé dans les programmes de l'enseignement primaire et a fortion secondarre, l'histoire de la Révolution, enseıgnée à la veille de la dernıère guerre mondıale dans la quası totahté des Unıversıtés Françaises, ne l'est plus aujourd'hui que dans quelques unes, mis à part la Sorbonne-Paris I, forteresse asslégée? - ainsi à Rouen, Tours, Dijon ou Aıx. Misère d'une pédagogie quı n'a pas été encouragée, comme en témoignent les difficultés à survivre de grandes collections documentaires (les Archives Parlementaires).

Avant de conclure prématurément, peut-être, convient-1l de nous interroger; et sans remonter au déluge, d'opérer le flash back indıspensable pour apprécıer la situation au vrai.

En termes de "Flash back": gloire et misère de l'historiographie révolutionnaire.

On peut parler d'un âge d'or de l'historiographie de la Révolution, si l'on se place à ce que Pierre Chaunu appelerait l'horí on 1900 , et ce que pour ma part j'aimerais qualifier d'époque jauressienne, quand Jaurès non seulement mène à bien l'ample saga de l'Histoire Socialiste de la Révolution écrivant dit-1l à la triple lumière de Michelet, de Marx et de 
Plutarque; introduisant en tous cas, ne fût-ce qu'à titre d'anticipation la pratique d'une approche résolument scientifique, mais aussi fait créer sous l'égide des Chambres Parlementaires la célèbre Commission de recherche et de publication de textes et de documents relatifs à l'histoire économique et sociale de la Révolution Française. Sur un chantier où se rencontrent des savants de tous pays - Minzes, Loutchisky, Kareiew, défricheurs de 1 'histoire agraire de la Révolution - s'inscrit

l'enchainement, alors, des grandes silhouettes de l'école française: Alphonse Aulard, premier occupant en 1886 de la chaire d'Histoire de la Révolution à la Sorbonne, poursuit avec Albert Mathiez un débat d'idées par héros interposés Danton contre Robespierre - lecture radicale contre lecture socialiste.

Mais cet aspect polémique ne masque pas l'extrême fécondité d'une recherche qui, de Mathiez à Lefebvre, à Soboul jusqu'à hier a posé les bases d'une école, diverse dans sa continuité, porteuse d'un discours progressivement élaboré sur la Révolution.

Une historiographie conquérante et sûre d'elle-même?

Sûre d'elle-même cette école jacobine? On l'a dit, et peut-être avec trop d'insistance. Conquérante, à coup sûr: depuis les chantiers de l'histoire politique, à laquelle ils ont su ne pas se tenir, ces maitres, à commencer par Mathiez (Mouvement social et vie chère sous la Terreur) pour poursuivre par Lefebvre (le Georges Lefbvre des Paysans du Nord sous la Révolution Française ou de $\mathrm{La}$ Grande Peur), pour culminer dans les Sans Culottes parisiens en l'an II d'Albert Soboul, ont élaboré une lecture sociale de la Révolution Française, introduisant progressivement sur la scène les masses rurales, puis urbaines, proposant le schéma explicatif d'une Révolution bourgeoise à soutien populaire, qui constituerait l'originalité de la voie révolutionnaire française, en un modèle où se réunifient les Révolutions bourgeoise, urbaine et paysanne - dont Georges Lefebvre avait dit la diversité.

Marxiste, ce modèle jacobin? Oui et non, adoptant certes le présupposé d'une mutation nécessaire, fondée dur le changement des structures sociales et des formes de production à la fin du XVIIIème siècle, autant et plus peut-être que sur l'évolution des idées. Mais suffisamment large et convaincant pour retenir l'adhésion d'historiens qui, de Marcel Reinhard à Jacques Godechot, pour ne citer que quelques uns restent plus jacobins que marxistes. Et l'on peut parler d'un véritable épanouissement dans les années 1950 à 1960 , quand les dernières années de Georges Lefebvre s'éclairent du rassemblement à Paris de toute une pléiade de chercheurs: A. Soboul, J.F. Suratteau, mass aussi venus de l'étranger, G. Rudé, A. Saitta, R. Cobb, K. Tovnesson, W. Markow ou K. Takahashi. La Révolution Française dirait-on n'a jamais attiré tant de monde: et c'est alors pourtant qu'Albert Soboul me tient les propos désabusés sur lesquels j'ai ouvert cette réflexion.

Soboul a raison: à cette époque déjà, la crise est ouverte.

\section{Un autre climat historiographique.}

A la fin des années 50, nous sommes au moment même du triomphe des Annales E.S.C., ces secondes Annales, animées par Fernand Braudel qui rédige alors son article célèbre sur La longue durée (1958). Pour lui comme pour tout le courant qu'il représente, la Révolution est de l'ordre des épiphénomènes, petite vague de l'histoire, reportée aux dérives de longue durée aux masses d'histoire lente qui constituent à son avis l'essentiel: ressortissant en somme de ce qu'il range
Pour lui comme pour tout le courant qu'il représente, la Révolution est de l'ordre des épiphénomènes, petite vague de l'histoire, reportée aux dérives de longue durée aux masses d'histoire lente qui constituent à son avis l'essentiel: ressortissant en somme de ce qu'il range avec un mépris non caché au rang de l'importun pathétique. 
avec un mépris non caché au rang de l'importun pathétique.

Triomphe de la longue durée, sur les chantıers de l'historre sociale, et bientôt de l'histoire de la civilisation matérielle, puis des mentalités ("Un temps plus long" selon R. Mandrou) et bientôt d'une anthropologie histonque qui tendra à se figer dans l'histoire immobile d' E. Leroy Ladurie.

La tentation fut grande pour nombre de chercheurs de s'investır dans la longue durée. Quı de nous n'y a pas cédé, sı peu que ce soit, et sans regrets? J'a1 mol même risqué dans La Mort et l'Occident de 1300 à nos jours, une fresque plurisécularre, sur un chantıer où la longue durée s'impose. Mass dans le domane précıs des études révolutionnarres, cette conjoncture défavorable allatt se doubler, dans ces années 1960, d'une attaque frontale contre les positions reçues.

\section{La grande attaque.}

Cette offensive est partie de plusieurs points: elle a trouvé dans les écoles anglo-saxonnes ses premiers champions (chez A. Cobban dans le Mythe de la Révolution, ou, Outre Atlantıque, chez G. Taylor Nor, capitalistic wealth at the origins of the French Revolution Mais ce courant a été très vite relayé en France où le livre de Françoıs Furet et Denıs Rıchet La Révolution Française, mit en 1965 le feu aux poudres. Si l'on résume en quelques propositions une série d'arguments, désormais entrés dans l'histoire de l'historiographie, l'attaque portait sur plusieurs thèmes, au demeurant très liés.

Sur les causes et sur l'interprétation sociale de la Révolution, là où Cobban avait dénié toute causalité sociale à un affrontement pour lui de l'ordre essentiellement du politique, d'autres contestaient la réalité de la réaction nobiliaire aux sources de la Révolution, et plus encore l'existence ou la consistance d'une bourgeossie véritable dans la France de la fin du XVIIIème siècle, soulignant qu'une part importante du capital, industriel et des entreprises tournées vers l'avenir étaient aux mains des nobles. . . (Taylor). Entre une noblesse progressiste, libérale et ouverte aux idées nouvelles, et la couche supérieure de la bourgeoisie, un consensus de fait n'exıstait-il pas dans le cadre des "elıtes" chères à D. Rıchet, et à quelques autres, et dans ces conditions la Révolutıon était-elle nécessaire, ne pouvait-elle être évitée ou stabilisée au stade d'un compromis réformiste, d'une monarchie constitutionnelle?

Etonnant retournements si l'on y réfléchit. ... moins de 20 ans plus tôt, en 1948, Daniel Guérın dans Bourgeois et Bras nus, la lutte des classes sous la Révolution Française, écrivant à la lumıère des théories de la Révolution permanente, avalt vu dans la dynamique révolutionnaire un mouvement trop tôt arrêté par la politique non sans machıvélisme de la bourgeoisie montagnarde, alors qu'll était porteur de son propre dépassement en termes de Révolution prolétarienne. Hypothèse aventureuse, que l'analyse concrète du contenu social de la sans-culotterie parisienne par Soboul devalt ruiner.

Désormals, dans la pensée des histortens que l'on commence à dénommer révisionnistes car 1ls se proposent de réviser de fond en comble les certıtudes reçues, c'est bien au contraire d'un mouvement trop loin poursuivi qu'il s'agit. Le compromis étart possible, on l'a frôlé en 1970 l'année heureuse; le dérapage de la Révolution Françaıse qui s'opère de 1791 à 94 est dû à l'intrusion incongrue des masses populaires urbaines ou paysannes, mobilisées sur la base de leurs revendications traditionnelles, en matière agraire ou de subsistances, sur un programme passéiste. 
Cette notion du dérapage de la Révolution entraîne la remise en cause de l'idée même d'un mouvement ascendant, de la Révolution bourgeoise à la Révolution démocratique de l'an II, où François Furet (dans son Catéchisme de la Révolution Française) voit des relents de finalisme, comme elle remet en question la théorie des circonstances, jusqu'alors admise, selon laquelle c'est pour faire face à la Contre Révolution intérieure, comme à la coalition des puissances monarchiques que la radıcalisation aurait dû se faure, fondée sur l'alliance momentanée, mais un temps efficace d'une partie de la bourgeoisie, et du mouvement populaire. La Révolution aurait-elle rêvé ces périls, créant des tıgres de papier, pour se livrer à un délıre dont elle s'intoxique elle-même? Un second discours du révisionnisme est déjà en germe dans ce farsceau de critiques.

Une nouvelle phase, une nouvelle donne?

Ma génération - celle des histonens qui ont atteint le demi siècle peu après 1980 - accuse sévèrement le choc, dans ses effectifs mêmes, de cette attaque, combinée à l'air du temps, du triomphe des Nouvelles Annales. C'est alors que nous avons pris conscience du recul de la place de la Révolution Française, non seulement dans la recherche, ou dans la pédagogie, mais dans une sensibilité et une culture qui lui devenaient étrangères.

Dirions-nous qu'une nouvelle phase commence avec 1968, ou la Révolution rêvée? L'argument serait trop facile sans doute. Et pourtant, c'est dans les années qui ont suivi ce mouvement qui se voulait une fête autant qu'une Révolution, qu'on a vu se multiplier les études sur la fête révolutionnaire: colloque de Clermont-Ferrand en 1974, ouvrages de Mona Ozouf et de Michel Vovelle en 1976. Comme fête, mais point seulement à ce titre l'événement révolutionnaire refait surface. La querelle des "jacobins" et des "révisionnıstes" qui semblait s'enliser dans une sorte de guerre de tranchées, souvent sans élégance, s'anime à nouveau pour le bien de la recherche.

\section{Dans le camp “jacobin”. . .}

Dans ce que nous appelerons, pour faire simple, le camp jacobin, les provocations, au bon sens du terme, reçues, ont conduit à d'utiles réflexions: ansi sur le concept de bourgeossie que de. . . Guizot à Lefebvre on n'avait pas suffisamment précisé, l'employant dans des acceptions, larges ou étroites parfois contradictorres. Des travaux comme ceux de Régine Robın (La France en 1789 Semur-en-Auxois) ont fortement contribué à éclaircir le problème en proposant les tratts d'une bougeoisie mixte, ou de transition caractérnstıque de cette phase, où le monde de la rente l'emporte encore sur celui du profit. C'est chez elle aussi qu'on peut chercher ainsı dans telle réflexion sur le concept de "liberté" dans le discours des Parlementaures lors des édits de Turgot sur la liberté des grains en 1776 - une analyse sans complaisance des ambiguités et des contradictions de la notion d'élites à la veille de la Révolution.

En même temps qu'Albert Soboul et ses élèves approfondissaient leurs recherches dans le champ de l'historre agraire (études sur le prélèvement seigneunal et la fin de la féodalité) comme urbaine (travaux sur le mouvement populaure parisien), d'autres chercheurs de même sensibilité (Michel Vovelle) proposaient une nouvelle lecture de l'histoire religieuse ou culturelle de la Révolution et s'efforçaient de poser les bases d'une histoire des mentalités révolutionnaires, annexant de nouveaux territoires à la recherche. . .

\section{Dans les rangs des révisionnistes. . .}

Entre temps, les choses ont également changé dans les rangs de l'école 
"révisionniste", dont le succés est incontestable non seulement en France mass dans le monde anglo-saxon, et dans toute une partie de l'Europe, à tel point que l'on peut se demander si une nouvelle vulgate n'est pas en train de se substituer à l'ancienne.

Elle est toutefors en renouvellement. Penser la Révolution Française, que $\mathrm{F}$ Furet publie en 1978, prolonge en les modıfiant singulièrement les propositions de 1965. Certes, ll revient sur la condamnation de la théorie des circonstances mais pour dire, citant Quinet "Non ce n'est pas la nécessité des choses qui a fait le système de la Terreur. Ce sont les idées fausses", ou même parlant en nom propre "le vra1 est que la Terıeur fait partie de l'ıdéologie révolutionnaire". Pour analyser ces sources endogènes de la dérive révolutionnaire, F. Furet s'appuie sur les historiens du XIXème siècie qu'll redécouvre parfois. sinon Tocqueville, ou - Quinet, qui n'étaient pas des oubliés, du moins Augustın Cochin, historien conservateur monarchiste du début du siècle, auquel il emprunte l'idée que la nouvelle sociabılité démocratıque et rousseauıste des loges maçonnıques et sociétés de pensée, fraie la voie à la reprise en main et à la confiscation totalitarre de la Révolution par la "machıne" jacobine ouvrant ce concept de souveraineté populaire dont 1 fait la "matrice du totalitarısme" (Penser la R.F., p. 232), estimant que "1789 ouvre une période de dérive de l'histoire".

La Révolutıon françarse reprend dans cette nouvelle lecture une cohésion certaine (on est loin du "dérapage") pursqu'elle acquiert le statut d'événement fondateur, mais, hélas, ce n'est pas en bien puisqu'elle se trouve contenir en germe les dérives totalitaires du XX'̀mc siècle. Par delà Cochın, Furet inscrit $1 \mathrm{c}$ sa réflexion en continuité avec celle de Talmon (Ortgins of the totaltarian democracy). Rousseau est en procès comme celur qui a porté les thèmes de volonté collective et de souveraineté nationale dont se sont
Seminário realizado na sede do IEA, em setembro de 1987. Da esquerda para a direita: Renato Janine Ribeiro, Ruy Coelho, Michel Vovelle e Carlos Guilherme Mota.

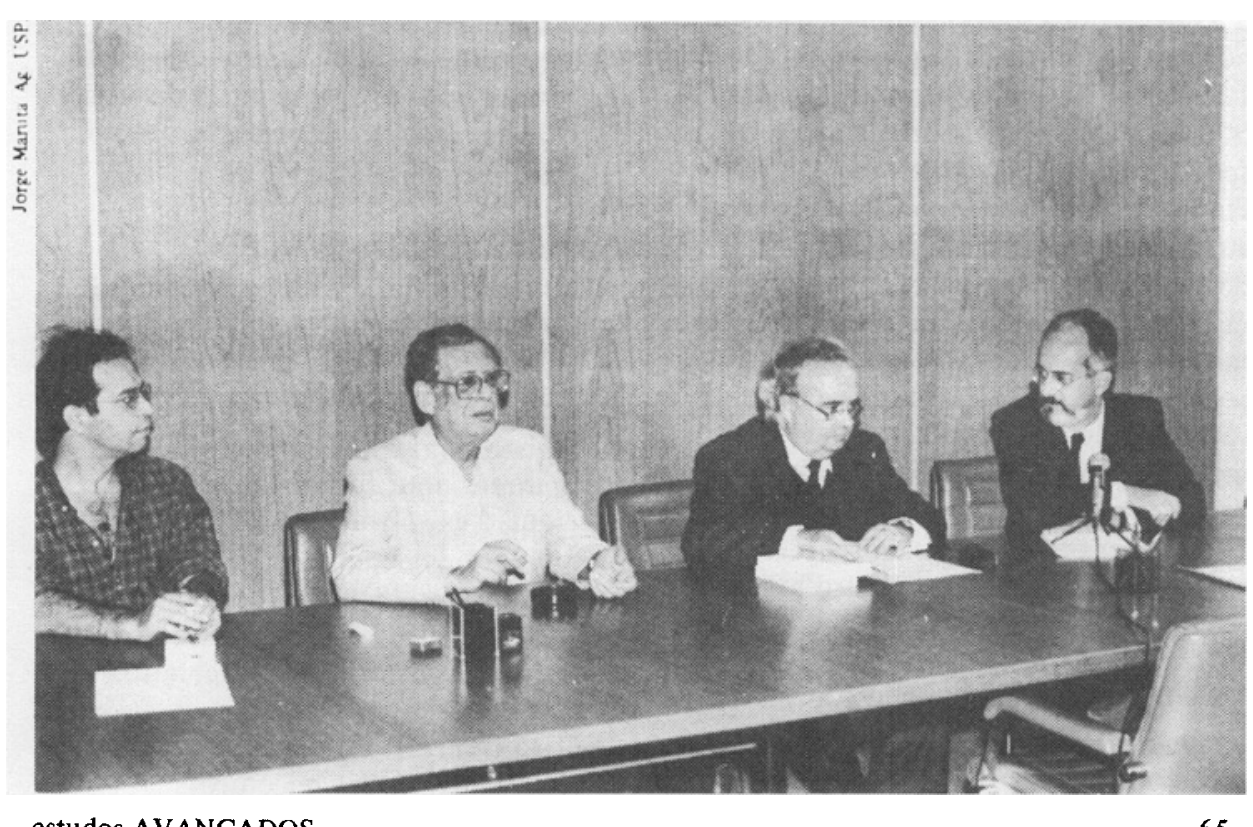

estudos AVANÇADOS 
nourris les jacobins: “C'est la faute à Rousseau" conclut Jacques Julliard qui partage ce point de vue (1986).

\section{Le réveil de l'histoire contre - révolutionnaire.}

Françoss Furet ne se reconnait pas, et l'a dit, dans le réveil récent, provoqué pour partie depuis deux ou trois ans par l'approche du bicentenaire d'une histornographie ouvertement contre révolutionnaire. A vra1 dire, avait-elle jamais disparu? Elle avait gardé ses positions fortes, de tradition depuis le XIXème siècle, à l'académie françasse (dans le sillage de Pierre Gaxotte) ou dans les bibliothèques des gares. Vieille chanson un peu fatiguée, elle a connu tout récemment un regain de vitalité remarquable. Petite monnare caricaturale des réflexions de François Furet, l'ımage d'une révolution totalitaire, antichambre du Goulag fait florès. La Révolution assimilée à la Terreur et au bain de sang devient le mal absolu. Toute une littérature se développe sur le thème du "génocide franco français" à partır d'appréciations souvent audacieuses du nombre des morts de la guerre de Vendée 128000,400000 . . . et pourquoi pas 600000 ? Certains historiens, sans être spécialistes de la question, ont mis, tel Pierre Chaunu, tout le poids de leur autorité morale qui est grande à développer ce discours de l'anathème, disqualifiant d'entrée toute tentative pour raison garder. Telle histoire tient beaucoup de place, en fonction des soutiens dont elle dispose, dans les médias comme dans une partie de la presse. Doit-elle nous cacher les aspects plus authentiques d'un chantier des études révolutionnaires aujourd'hui en plein réveil?

\section{Un chantier en plein réveil.}

Dresser un bilan au vrai des chantiers actuels de la recherche sur là Révolution est une tâche facilitée par la conjoncture même du bicentenaire qui, en stimulant la demande, fait mieux apparaitre les traits de la production. Les comptes rendus que dresse périodıquement dans la Revue historique Jacques Godechot, le flux plus simplement de l'édition, fournissent des tests assez sûrs. Par alleurs, le bulletin publié depuis 4 ans par la Commission de recherche historique (C.N.R.S.) pour le bicentenaire de la Révolution, recensant programmes de recherches et colloques qui en sont l'expression, offre une couverture plus fiable encore des tendances de la recherche, telle qu'elle se pratıque aujourd'hus sur le chantier, en France et dans le monde.

Ne nous cachons pas que certans de ces indices sont à interpréter. aınsi la profusion éditoriale des dernières années révèle-t-elle aussi des phénomènes de mode, des engouements entretenus par les médias, autant et parfors plus qu'elle ne reflète le mouvement authentıque de la recherche alors même que les collections érudites et publications savantes de textes et de documents (les Archives

Parlementaires) ont de la peine à survivre. On touche $1 \mathrm{c}$ le problème de la distorsion entre le discours des doctes et celui qui est porté par les médias: nous y reviendrons.

Cette précaution prise, il est néanmoins loisible de tenter un tableau ou un bilan en marche.

\section{Redécouverte du Politique.}

L'historiographie actuelle de la Révolution, pour suivre un parcours classique d'exposition, a vu s'arrêter le mouver:ent de déclin de l'historre politique, sensible dans toutes les branches de l'histoire sous l'influence de l'École des Annales. Une réévaluation du politique s'est opérée $1 c i$, significativement. En ferons-nous une des conséquences des relectures 
"révısıonnistes" apportées par François Furet? Il est vra1 qu'elles définıssent une approche très spécifique du politıque, dans le cadre d'une histoire conceptualisée selon l'expression de l'auteu, qui sans trop recourir aux apports d'une recherche de terrain que F. Furet n'apprécie guère, se tourne plutôt, pour ennichir son modèle explıcatif vers la redécouverte régressive des précédents histonographiques s'attachant à Tocqueville, Quinet, Marx, et bien sûr Augustın Cochın. Cette école n'est point la seule toutefors à ouvrer dans le domaine du politique révolutionnarre: une partıculıère attention doit être portée à cet autre courant où se réalıse, à partır de l'analyse du discours, menée par des lexicologues qui sont aussi des historiens, une approche des contenus - qu'il s'agisse du discours jacobın, ou de celui du mouvement populaire et de ses porte parole - Hébert ou d'autres -. Les études de Jacques Gullhaumou ou Annie Geoffroy, mais d'autres encore, sont à cet égard très significatives.

D'où qu'on vienne, la convergence se fait en ce domaine sur un certain nombre de questions tests: et celle du jacobınisme (Claude Mazauric "Jacobınısme et Révolutıon" (1984) est bien le type même de ces questions centrales, où se cristallise le débat sur le sens même de la Révolution.

\section{L histoires des homnıes: ambiguités de la biographie.}

$\mathrm{Au}$ coeur de cette redécouverte du politique, l'approche biographique tient une place ambigue. On l'eut dit hier, à bon droit, en déclin, et songeant aux grands débats du début du siècle Danton contre Robespierre, Aulard contre Mathiez - nous aurions pu écrire à l'instar de Lucien Febvre "sur une histoire qui n'est plus la nôtre". Puis voic1 que, tout récemment, sur un chantier des biographies révolutionnaires qui sembalit surtout réservé désormais aux historiens académiques, un retour s'est opéré: Saint Just, Danton, Mirabeau, Madame Roland, Lucile et Camille Desmoulins retrouvent de nouveaux biographes, souvent de qualité, de façor. assez révélatrice. Le trait n'est pas spécifique du chantier révolutionnaire car ces retour de la bicgraphie qui ont été analysés ( $\mathrm{n}$. spécial de la revue Espaces-Temps, 1986) sont un des traits généraux d'une historiographie d'aujourd'hui en mal d'identifications et de personnalisation. On cherche ses héros comme on cherche ses racines. Du moins, à côté des grandes figures, I'histoıre révolutionnaire se prête-t-elle aussi à valoriser les "études de cas", qui ont contribué à renouveler la conception même de la biographie en se penchant sur les héros anonymes ou semi-anonymes, dont l'aventure peut être aussi éclairante que celle des premiers rôles: on songe au maítre vitrier Ménétra dont le "Journal" a été étudié par Daniel Roche*. Et j'ai moi même "fait parler" deux de ces anonymes en Révolution, le maítre menuisier d'Aix-en-Provence, Joseph Sec, qui se révèle (dirons-nous tout entier?) dans son tombeau cénotaphe maçonnique et jacobin, comme le poète Thédore Désorques, auteur de l'Hymne à l'Etre Suprême du 20 prairial an II, exemple à la limite de l'artiste en Révolution.

Un détour actuel de la biographie prend dans le cadre des études revolutionnaires une importance particulière: c'est celui qu consiste à suivre les processus d'héroisation, ou de fabrication posthume des grandes figures révolutionnaires, au sein même de l'aventure collective: ce travail de

" "Journal de ma vie" de Ménétra. 
l'imaginaire a été suivi dans "la mort de Marat" de façon tout à fait exemplaire par une équipe de chercheurs dans une étude interdisciplinaire.

\section{Histoire sociale, histoire des masses.}

In reste que les retours du politique, pas plus que ceux de la biographie ne sauraient masquer le pords essentiel de cette histoire sociale autant et plus que politique des masses en Révolution qui demeure bien, dans la suite de 1'historiographie jacobine l'objectif essentiel.

Cette histoire a ses chantiers et ses projets. Le chantier parisien, défriché successivement par Marcel Reinhard sous l'angle sociologique et démographique, puis par Albert Soboul et ses élèves au regard des formes du mouvement populaire, est fort loin d'être épuisé: on espère pouvoir en 1989 présenter une synthèse, sinon définitive du moins reformulée de l'ensemble de ces travaux. Puis il convient, si l'on me passe l'expression de "déparisianiser" l'histoire de la Révolution Française, en prenant possession renforcée de l'espace national. C'est le but que se poursuit la constitution en cours à l'Institut d'Histoire de la Révolution Française (Paris I) d'un centre de documentation national sur micro-fiches, rassemblant les données actuellement dormantes ou stériles des mémoires de maîtrises ou des thèmes, tant provinciaux que parisiens. Dans le même sens, on travaille à mettre sur pied un Atlas Historique de la Révolution Française (projet conjoint de 1'E.H.E.S.S. et de 1'T.H.R.F.). A ce programme formulé dans l'absolu, la réponse des régions est vive: les travaux se sont multipliés, tant sur l'Ouest révolutionnaire et contre-révolutionnaire (Colloque de Rennes, 1985 Les Résistances à la Révolution) que sur le Midi provençal et languedocien le Dauphiné, la région du Nord: énumération forcément incomplète des chantiers en cours à la veille du bicentenaire. Dans ces cadres régionaux, les différentes branches de l'histoire sociale révolutionnaire témoignent d'un dynamisme inégal, alors que l'histoire économique, malgré des travaux brillants et récents (D. Woronoff: l'industrie sidérurgique, L. Bergeron: banquiers et hommes d'affaires, Bruguière sur les spéculateurs) reste trop confinée à un cercle de spécıalistes, comme peùt être 1'histoire démographique, au lendemain du coup de fouet que lui avalt donné Marcel Reinhard.

\section{L histoire rurale n'est pas morte.}

L'histoire rurale n'a pas régressé, tant dans l'approche des structures, ou Albert Soboul a animé jusqu'à sa mort une recherche sur l'étude du prélèvement seigneurial et son éradication sous la Révolution (thèses de J.N. Luc sur "L'élimination des droits féodaux en Charente Maritime" et de Guy Lemarchand sur "La fin du féodalısme dans le pays de Caux"). Quant à l'approche de la dynamique sociale, à partir des mouvements paysans sous la Révolution - ainsi dans la grande crise du printemps et de l'automne 1792, mais aussi bien dans le mauvais gré généralisé contre le racahat des droits féodaux quı touche certaines régions en 1790 - c'est un chantier en pleine activité à partir des travaux de A. Ado (malheureusement trop mal connus encore), de $M$. Vovelle et de J. Nicolas qui entreprend une enquête d'ampleur nationale (Colloque tenu à l'Université de Paris VII en 1984 sur les émotions populaires à l'époque moderne).

\section{Histoires urbaines.}

Les histoires urbaines - sociologie et mouvement social intimement mêlées vont leur train, en continuité avec les apports de Soboul, de Rudé (enfin
Il reste que les retours du politique, pas plus que ceux de la biographie ne sauraient masquer le poids essentiel de cette histoire sociale autant et plus que politique des masses en Révolution qui demeure bien, dans la suite de 1 historiographie jacobine l'objectif essentiel. 


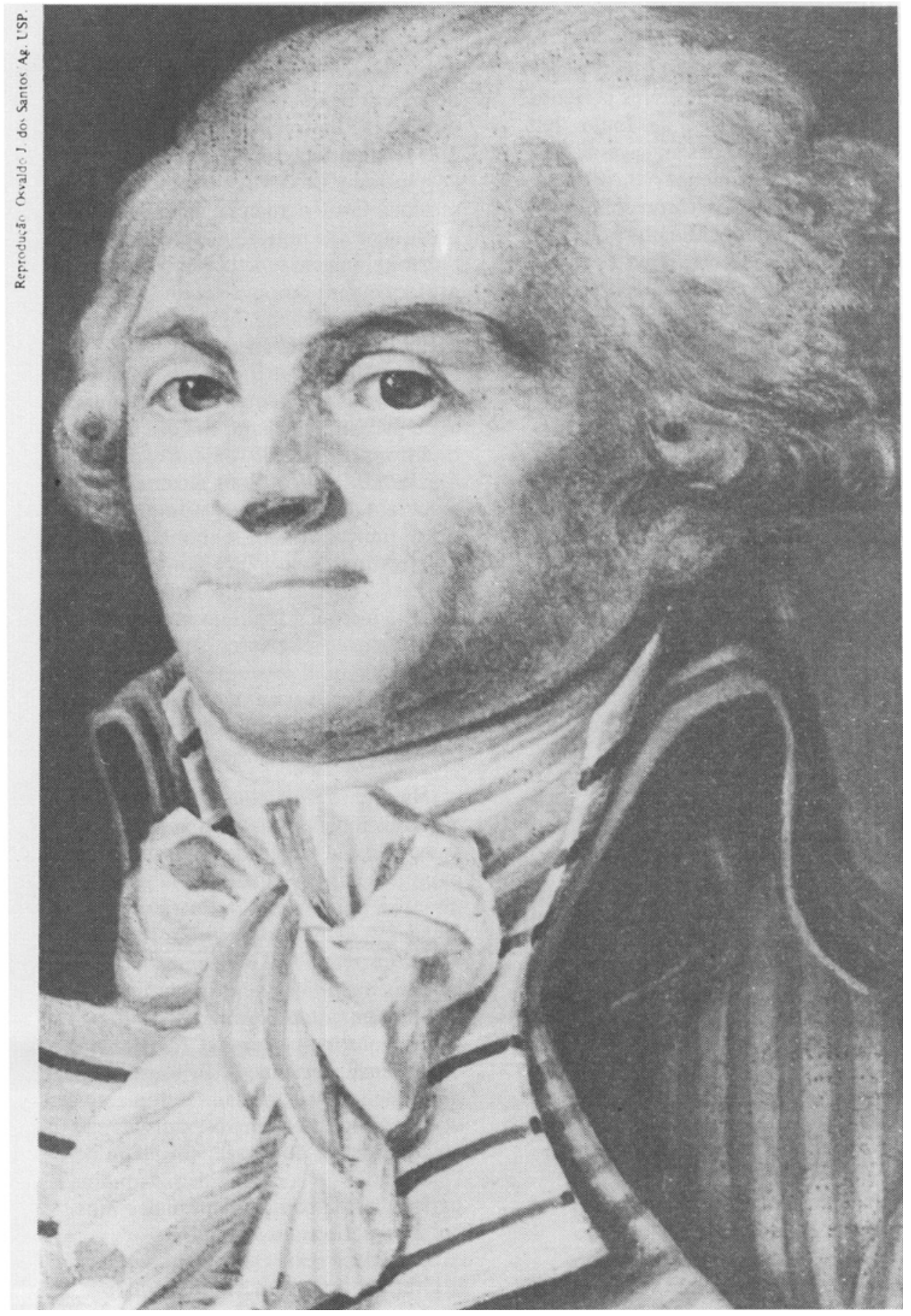

Robespene 
traduit en français. .. après 25 ans pour ses "foules révolutionnaires"). On explore systématiquement de grands chantiers ouverts: citons sans prétendre à l'exhaustivité, l'étude des foules, qui débouche sur celle des langages et des gestuels de la violence (B. Conein sur Les massacres de septembre 1792). De même une étude sociologique du fédéralisme dans ses différentes formes est-elle en cours (Voir la récente publication collectives sur le Fédéralisme jacobin, 1986). Dans cette démarche, après les groupes de la sans culotterie étudiés par Soubol (Paris) ou Vouvelle (Marseille), un investissement se tourne légitimement, à nouveau, vers les attitudes et stratégies des bourgeoisies en Révolution, à partir de la prosoprographie des élites et notables municipaux.

Enfin cette histoire sociale rejoint l'histoire politique dont elle est indissociable dans le vif courant de curiosité qui se porte sur les contre révolutions paysannes, sur la façon et les raison du basculement de toute une partie des masses paysannes dans l'hostilité, éventuellement armée au nouveau régime: le colloque déjà cité sur les Résistances à la Révolution (RENNES, 1985) est à cet égard exemplaire. Une série d'hypothèses de travail ont été élaborées depuis la grande thèse de référence de Paul Bois sur "Les paysans de l'Ouest", dont les conclusions sont parfois aujourd'hui contestées (Roger Dupuy, D. Sutherland), dans un débat d'idées qui n'a nen à voir avec les misérables polémiques sur le génocide franco-français.

\section{En passant par le religieux:} l'emphase sur le culturel.

Reste qu'à travers l'analyse des nouvelles publications, comme des chantiers de la recherche et rencontres scientifiques, s'inscrit avec unẹ particulière netteté une emphase mise sur le culturel, puis le mental. Peut-on parler d'une "derive" sur l'imaginaire au détriment de l'étude des conditions objectives? Pour n'être pas spécifique que de la période révolutionnaire, l'évolution y est particulièrement sensible. Cette démarche ne va pas sans provoquer une nouvelle approche de l'histoire religieuse de la Révolution, profondément renouvelée: on s'est attaqué au problème de la déchristianisation, événement traumatique qui introduit aux formes de la religiosité proprement révolutionnaire, et de libération à chaud des disciplines traditionnelles (M. Vovelle, Religion et Révolution, 1976). Tout récemment en réouvrant le dossier des attitudes du clergé français devant le serment constitutionnel de 1790, Timothy Tackett (Clergé, Révolution Nation, 1986) a insisté autant que sur ses conséquences religieuses, sur la rupture irréversible qu'il provoque, dans tout l'espace française, au niveau des options pour ou contre la Révolution: événement structurant de grand avenir.

Mais les aspects plus spécifiquement culturels focalisent aussi nombre de recherches: la table rase révolutionnaire, exprimée par le vandalisme, la politique directive notamn jent em matière de langues et de patois (D. Julia, J. Revel, M. De Certeau: Une politique de la langue), ne représentent qu'un pan de ce que plusieurs ont exprimé en termes de "Révolution culturelle" (S. Bianchi), on se penche également sur toute une politique innovatrice dans le domaine des sciences et des techniques, dans les arts où la naissance du musée, corollaire de l'émergence de la notion de patrimoine national est le complément dialectique des destructions du vandalisme.

Enfin l'ex trême créativité dans la littérature, dans la musique, comme dans l'expression graphique et 
iconographıque d'une période qu'on a dit à tort stérile suscite toute une série de découvertes de chantiers jusqu'alors peu prospectés (M. Vovelle, La Révolution Française, Images et Récits, 1986).

\section{Du culturel aux mentalités.}

De la culture aux mentalités, la transitıon semble a1sée, et comme naturelle. Malgré les précédents célèbres (La Grande peur, de Georges Lefebvre), les approches nouvelles d'histoire des mentalıtés ont rencontré quelques difficultés à s'imposer sur un chantier où planait l'ombre de Taine et de ses élèves. C'est toutefors chose farte aujourd'hu1 (Michel Vovelle, La mentalité révolutionnaire) alor même que l'on s'interroge sur les visages de cet "homme nouveau" que la Révolution Française a entrepris de façonner, prıs comme le disait G. Lefebvre entre les pulsions contradictorres de l'espérance et de la peur, inséré dans les nouvelles sociabilıtés, du club à la fête. . .

Au cœur même de la vie des hommes, la Révolution assume ainsı pleınement ce rôle d'événement fondateur, aux retombees de longue durée: celles mêmes que tout un courant de recherches entreprend d'analyser.

\section{L'image projetée de la Révolution Française.}

Les avatars des idées force, et des Enfin l'ex trême créativité dans la littérature, dans la musique, comme dans l'expression graphique et iconographique d'une période qu'on a dit à tort stérile suscite toute une série de découvertes de chantiers jusqu'alors peu prospectés.
A gulhon sur les expressions allégoriques de la Républıque, sous la forme des "Marianne" du XIXème siècle*. Mats disons plus largement que la très forte mobilisation internationale qui s'effectue au plan scientıfique à l'approche du bıcentenaire vise très naturellement à analyser ces aventures posthumes de la Révolutıon Françaıse, à travers la diffusion de ses ıdées force dans les différents pays. La réponse multiple des historiographies nationales en témoigne.

\section{En termes de conclusion: triomphante ou menacée.}

Pour rapide qu'il soit, le tableau auquel nous venons de nous livrer ne saurait confirmer l'impression de sclérose, de déclın ou de répétıtıvité que le dictıonnaire des idées reçues nous ava1t retransmis. L'historiographie de la Révolution Françaıse est en pleın réveil. On ne saurait plus désormass parler aujourd'hui d'une lecture hégémonique en ce domaine, marqué par une explosion tous azimuths, et c'est sans doute un bien. Reste que cette histonographie trahit aussi son désarroi, entre les scrupules d'une histonographie "jacobıne" qui reprend lentement confiance après avour été la cible de toutes les attaques, le malaise d'une historiographie "révisionniste" qui a peut-être épuisé sa nouveauté sainement provocatrice, et se trouve confrontée au réemploi, vulgarısé et travestı par une troisıème école, que nous dirons de "l'anathème" d'idées forces lancées hier sur les "derıves" Inévitables de la Révolution. Quant à ce troisième groupe, si sa place sur le plan scientifique n'est pas grande disqualıfié par un recours à la polémıque qui luı enlève sa

\footnotetext{
* Les "lieux de mémoire" que P'zere Nora a entrepris de recenser dans un ample ouvrage collectıf, nous introduit directment à ce chantier.
} 
crédıbılité - il n'en ba pas moins l'estrade avec assurance, pour quelques temps.

Cela ne facilite guère le nécessaure traval de rattrapage, ou de réapprivoisement, dans le public cependant très désireux de découvrir, d'une image altérée tant par la perte de la mémoire collective que par le recul des connarssances. Il serait très dommageable que le bicentenaire voie s'ouvrir ains1 les branches des ciseaux, entre une recherche historique active et ouverte, et un discours véhiculé par les médias sur les thèmes les plus rebattus d'une tradition très largement contre-révolutionnaire, naturalısant l'image d'une Révolution exclusıvement vue à travers ses aspects sanglants et destructeurs.
Reste que dans cette situation ouverte, les raisons d'espérer ne manquent pas. $\mathbf{L a}$ Révolution n'est pas "terminée". elle reste bien l'un des tests discriminants les plus fortement marqués dans l'imaginare collectif des français. Hors de France, elle suscite une remarquable convergence d'intérêts, une sympathie collectıve quı s'étonne souvert des états d'âmes des français eux-mêmes. On peut espérer que cette convergence de demandes, nationales, provinciales et internationales trouvera son écho en 1989 dans la tenue du Congrès Mondial prévu sur "L'ımage de la Révolution Françaıse". Il est en tous cas de la responsabilıté des historiens de se montrer, comme on disait sous la Révolution, à la hauteur des circonstances. C'est un rendez-vous historique à ne pas manquer.

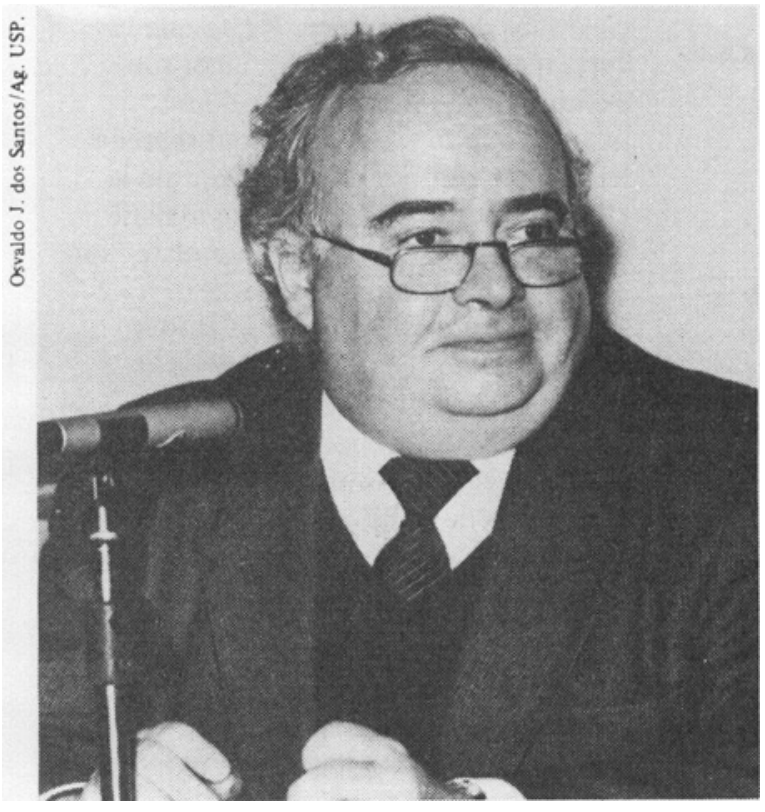

72

\section{Michel Vovelle}

Michel Vovelle, 54, é professor de Históna da Revolução Francesa na Universidade de Parıs I (Sorbonne), diretor do Instituto de Histórıa da Revolução, presıdente da Comıssão do Bıcentenáno da Revolução Francesa e um dos mais sıgnificatıvos historiadores contemporâneos. É o grande investigador da História das Mentalidades e das Ideologias, tendo pesquisado, entre outros temas, a questão das mentalidades coletıvas e as atıtudes que as pessoas têm diante da morte (e da vida). Autor de numerosos livros, onde podem ser destacados Mounr autrefols (Gallimard, 1974), Reltgion et Révolutıon la déchristianisation de l'an II (Hachette, 1976), La Mort et l'Occident de 1300 ì nos jours (Gallumard, 1983), Theodore Desorgues ou la désorgantsation (1763-1808) (le Seuil, 1984), La Mentalté Révolutionnatre (Messidor, 1985), e Idéologzes et Mentalités (Maspéro, 1982), recentemente traduzıdo para o português pela Eđitora Brasiliense.

estudos AVANÇADOS 